\title{
A New Method for Phase Contrast Tomography
}

\author{
A. Groso, R. Abela, C. David and M. Stampanoni \\ Paul Scherrer Institut, CH-5232 Villigen PSI Switzerland
}

\begin{abstract}
We report how a method which requires a simple experimental setup, in principle equivalent to the one for doing classical absorption tomography, can yield phase information in three dimensions in only one single step. As an alternative to two steps approaches (phase retrieval and reconstruction of the object function applying a conventional filtered backprojection algorithm), A.V. Bronnikov suggested an algorithm which provides direct 3D reconstruction of the refractive index from the intensity distributions measured in a single plane (pure phase object) and from the intensity distributions measured in two planes (mixed phase-amplitude object). We adapted the original algorithm in order to retrieve the $3 \mathrm{D}$ reconstruction of the refractive index from the intensity distributions measured in a single plane also for the case of mixed weakly absorbing-phase object. The results show that the contrast is increased, while keeping dose minimal and spatial resolution equivalent to the conventional absorption based technique.
\end{abstract}

Keywords: x-ray imaging, phase contrast, tomography

PACS: $81.70 . \mathrm{Tx}, 42.30 . \mathrm{Wb}$

\section{INTRODUCTION}

Conventional $\mathrm{x}$-ray computed tomography (CT) is based on the difference in x-ray absorption by different materials and is nowadays a standard technique with spatial resolution around one micron [1-4]. On the other hand, a wide range of samples studied in materials science, biology and medicine show very weak absorption contrast, yet producing significant phase shifts of the x-ray beam. The use of phase information can therefore increase the contrast substantially.

As far as phase tomography (quantitative 3D reconstruction of the phase or the refractive index from 2D phase images) is concerned, several attempts were made by using interferometric [5-6] and non-interferometric phase retrieval methods [7-13]. All these methods are based on a two step approach: first, the projections of the phase are determined in the form of Radon projections and then the object function, i.e. the refractive index $\delta$, is reconstructed applying a conventional filtered backprojection algorithm.

Alternatively, the reconstruction algorithm suggested by Bronnikov in his theoretical works $[14,15]$ presents an approach which requires no intermediate step of $2 \mathrm{D}$ phase retrieval and provides a direct $3 \mathrm{D}$ reconstruction of refractive index from the intensity distributions measured in a single plane (pure phase object) and from the intensity distributions measured in two planes in the case of mixed phase-amplitude object.

In this work we applied the quantitative phase contrast CT method presented in $[14,15]$ to real experimental data. We adapted the original algorithm in order to retrieve the $3 \mathrm{D}$ reconstruction of the refractive index from the intensity distributions measured in a single plane also in the case of mixed weakly absorbing-phase object. In this way the experimental setup can be kept very simple and the radiation damage is kept to the minimum.

\section{THEORETICAL BACKGROUND}

Let consider a monochromatic plane wave with wavelength $\lambda$ that propagates along the positive z-axis and that impinges upon a thin mixed phase-amplitude object, which is characterized by the linear absorption coefficient $\mu\left(x_{1}, x_{2}, x_{3}\right)$ and the real part of the index of refraction $\delta\left(x_{1}, x_{2}, x_{3}\right)$. The intensity distribution $I(x, y)$ at a distance $z=d$ and angle of rotation $\theta$ can be expressed by [9]: 


$$
I_{\theta, z=d}(x, y)=I_{\theta, z=0}(x, y)\left[1-\frac{\lambda d}{2 \pi} \nabla^{2} \phi_{\theta}(x, y)\right]
$$

with $\phi_{\theta}(x, y)=-\frac{2 \pi}{\lambda} \int_{\Re^{2}}\left[\delta\left(x_{1}, x_{2}, y\right) \times \widetilde{\delta}\left(x-x_{1} \cos \theta-x_{2} \sin \theta\right)\right] d x_{1} d x_{2}$ being the phase function of the object and

$\widetilde{\delta}$ denoting the Dirac function. Expression (1), which corresponds also to the Transport of Intensity Equation (TIE) in its simplified form [6-11], is valid (i) in the near-field Fresnel region $\left(d<<a^{2} / \lambda\right.$, where $a$ is the transversal size of the smallest structure in the object) and (ii) for a mixed phase-amplitude object with weak and almost homogeneous absorption (i.e. $\partial \mu / \partial x, \partial \mu / \partial y \approx 0$ ).

The goal of quantitative phase tomography is to reformulate Eq.1 to obtain $\delta\left(x_{1}, x_{2}, x_{3}\right)$ from the knowledge of $I_{\theta, z=d}(x, y)$ for $\theta \in[0, \pi]$. Expressing Eq.1 as $\nabla^{2} \phi_{\theta}(x, y)=-\frac{2 \pi}{\lambda d} g_{\theta}(x, y)$ with $g_{\theta}(x, y)=\frac{I_{\theta, z=d}(x, y)}{I_{\theta, z=0}(x, y)}-1$, applying then the 3D Radon transform (denoted by the symbol $\wedge$ ), and calculating finally the second derivative with respect to the variable $s=x \sin \omega+y \cos \omega$, one gets: $\frac{\partial^{2}}{\partial s^{2}} \hat{\delta}(s, \theta, \omega)=-\frac{1}{d} \hat{g_{\theta}}(s, \omega)$.

Above expression is a theorem which states that from the 2D Radon transform of the measured value $g$, one can directly find the 3D Radon transform of $\delta$ [15]. The computation of the two dimensional Radon transform and its backprojection can be combined into a single step and, defining $q(x, y)=\frac{|y|}{x^{2}+y^{2}}$, one obtains:

$$
\delta\left(x_{1}, x_{2}, x_{3}\right)=-\frac{1}{4 \pi^{2} d} \int\left(q * * g_{\theta}\right) d \theta
$$

where the stars indicate a 2D convolution. This convolution integral can be computed in the Fourier domain by taking the two dimensional Fourier transform. In the Fourier domain, equation $q(x, y)=\frac{|y|}{x^{2}+y^{2}}$ has a low-pass filter form given by [15]:

$$
q(\xi, \eta)=\frac{|\xi|}{\xi^{2}+\eta^{2}}
$$

Expressed in this form (Eq. 2), the approach becomes very interesting since in case of a pure phase object (i.e. $\left.I_{\theta, z=0}(x, y)=1\right)$ the 3D distribution of the refractive index can be recovered from only one single tomographic data set. This is a significant improvement since (a) the experimental setup can be kept extremely simple (it is actually the same as for standard, absorption based tomography) and (b) the radiation damage is kept to the minimum, which is of special importance for biological specimens.

\section{RESULTS AND ANALYSIS}

In a previous work [16] we simulated intensity projection data, implemented the aforesaid reconstruction algorithm and successfully reconstructed the object refractive index. In a further step, the original algorithm $[14,15]$ has been used to reconstruct phase tomograms from experimental data sets.

Experiments were performed at the Tomography Station of the Materials Science beamline at the Swiss Light Source [3]. As already pointed out, the experiment setup is equivalent to the one for standard absorption tomography. Nevertheless, two additional conditions have to be satisfied: (i) sample-detector distance (SDD) is now increased from the minimum $\mathrm{SDD}=0$ used in absorption tomography, to the near field Fresnel region where $S D D<<a^{2} / \lambda$ ( $a$ is the transversal size of the smallest structure in the object and $\lambda$ is the $\mathrm{x}$-ray wavelength) and (ii) the photon energy was set to the maximum attainable at the experimental station $(25 \mathrm{keV})$ in order to satisfy as much as possible weak and almost homogeneous absorption condition $(\partial \mu / \partial x, \partial \mu / \partial y \approx 0)$.

As a test, one millimeter thick polyacrylate, starch and cross-linked rubber matrix sample was investigated. There is a rather weak contrast when absorption tomography is used [Fig. 1a)]. In order to apply phase tomography method in pure phase object approximation $\left(I_{\theta, z=0}(x, y)=1\right)$ a single data set $\left(I_{\theta, z=d}(x, y)\right)$ of 721 angular projections at $d$ of $80 \mathrm{~mm}$ and $25 \mathrm{keV}$ photon energy was acquired. Fig. 1b) presents a slice through the sample reconstructed using Eq. 
(3) and (2). Even though the absorption level for this object is calculated to be in the range of only $3 \%$, pure phase object condition is not satisfied - the image is severely corrupted by the residual absorption artifact (see cap in the center of Fig. 1b)) meaning that in principle also intensity measurement at 'zero' distance $\left(I_{\theta, z=0}(x, y)\right)$ is required.
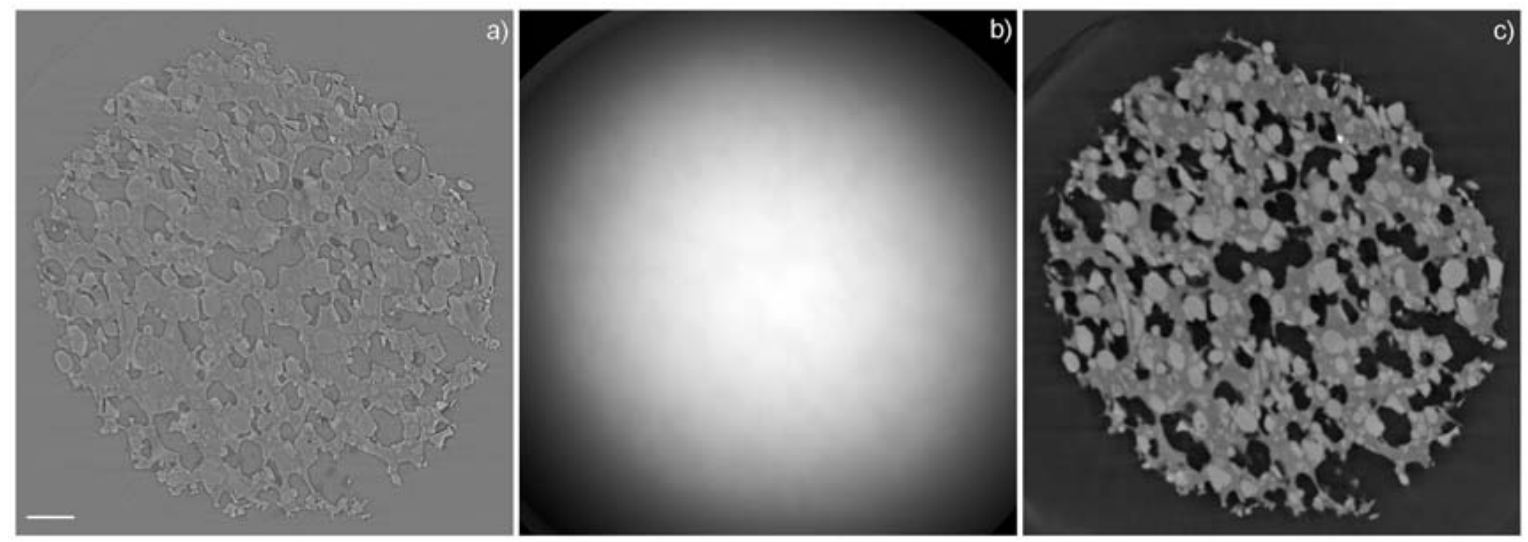

FIGURE 1. Tomographic reconstruction of a polymer sample: (a) absorption contrast, (b) phase contrast obtained with uncorrected filter, Eq. 3 (c) phase contrast obtained with correct filter, Eq. 4. The length of the scale bar is $100 \mu \mathrm{m}$.

We have adopted another approach - the reconstruction artifacts (observe Fig. 1b)) are corrected by amending the original method [15] leading to a modified version of Bronnikov's algorithm (MBA). The correction consists of adding, in the denominator of the low-pass filter given in Eq. (3), an absorption dependent correction factor $\alpha_{\text {exp }}$. Consequently, the new filter has the following form:

$$
q(\xi, \eta)=\frac{|\xi|}{\xi^{2}+\eta^{2}+\alpha_{e x p}}
$$

The values of $\alpha_{\text {exp }}$ to be used are found by using a semi empirical (simulations-experiment) approach. The details are given in [17]. Applying the MBA method, we reconstructed the same data set (rubber matrix) and the (artifacts free) result is presented in the Fig. 1c).

In order to validate our approach, we have compared its results with the ones obtained using an established differential phase contrast method (DPC) [6]. To make a direct evaluation, experiments using the two techniques have been performed on the same samples and with the same detector effective pixel size (1.4 microns).
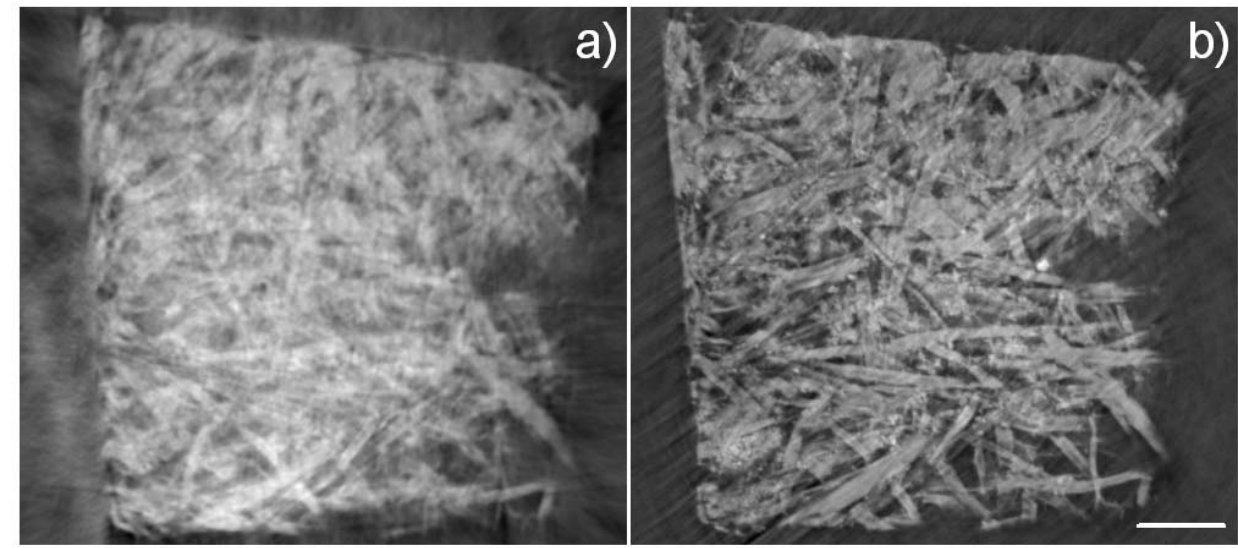

FIGURE 2. Validation of the MBA method: Phase tomographic reconstruction of paper obtained (a) using DPC and (b) using MBA. The length of the scale bar is $100 \mu \mathrm{m}$.

Figure 2a) presents the result obtained using the DPC technique. 501 angular projections with 4 phase steps for every angular projection were acquired at a photon energy of $17.5 \mathrm{keV}$. Total scan time was $6.5 \mathrm{hrs}$ and total exposure time $3.3 \mathrm{hrs}$. For every angular position the projection of the phase is determined and then the phase in 3D is reconstructed using conventional filtered backprojection algorithm. Figure $2 b$ ) shows the same sample reconstructed with the MBA technique. One tomographic data set (501 angular projections) was acquired at SDD of 
$22 \mathrm{~mm}$ (using $25 \mathrm{keV}$ photon energy) and reconstructed applying Eq. (4) and Eq. (2). Total scan time was 1.5 hrs and total exposure time 50 minutes.

\section{DISCUSSION}

We have demonstrated that the 3D distribution of the phase (refractive index) of a weakly absorbing object can, alternatively to the combined phase retrieval-backprojection methods (two steps) presented up to now, be directly reconstructed from a single tomographic data set (one step). In order to reduce the residual absorption artifact the method presented in Ref. [15] has been amended leading to what we named the Modified Bronnikov Algorithm (MBA). The results show that the contrast is increased, while keeping experimental setup simple and radiation dose minimal.

The MBA was validated using the Differential Phase Contrast Imaging technique. In general, the two methods are complementary. The full 3D phase imaging approach (MBA) is experimentally simple, very fast and it is ideally suited for small objects when resolution around 1 micron is needed. Deposited dose is equivalent (or lower, since higher photon energy is used) to the one deposited in conventional tomography. The DPC is more demanding in terms of instrumentation and acquisition time (therefore also deposited dose) but it is more sensitive and can be scaled up to large fields of view.

Artifacts/non perfect reconstruction using the MBA method are due to the fact that estimated absorption (prior to the filtering step) is approximated as constant over entire object. Additionally, detector sensitivity/noise and beam instability also induce low frequencies problem, deteriorating the reconstruction quality.

Future developments will focus on the fine tuning of the filtering step and on the streamlining of the whole process in order to allow high-resolution and high throughput imaging of soft biological and materials science specimens.

\section{ACKNOWLEDGMENTS}

We are grateful to A. Bronnikov and O. Bunk for fruitful discussions and to R. Rudolf for providing the samples. The help of P. Schneider, S. Linga and R. Müller in performing simulations and F. Pfeiffer in DPC reconstructions is gratefully acknowledged. Part of this work was supported by the Swiss National Science Foundation (FP-62058097.99 and PP-104317/1).

\section{REFERENCES}

1. U. Bonse and F. Bush, Prog. Biophys. Molec. Biol., 65, 133-169 (1996)

2. B.A. Dowd, G.H. Campbell, R.B. Marr, V.V. Nagarkar, S.V. Tipnis, L. Axe and D.P. Siddons, Developments in X-Ray Tomography II, Proc. SPIE 3772, 224-236 (1999)

3. M. Stampanoni, G. Borchert, P. Wyss, R. Abela, B. Patterson, S. Hunt, D. Vermeulen P. Rüegsegger, Nucl Instrum Meth. A 491 (1-2), 291-301 (2002)

4. T. Weitkamp, C. Raven and A. Snigirev, Developments in X-Ray Tomography II, Proc. SPIE 3772, 311-317 (1999)

5. A. Momose, Nuclear Instruments and Methods A 352, 622-628 (1995)

6. T. Weitkamp, A. Diaz, C. David, , F. Pfeiffer, M. Stampanoni, P. Cloetens, E. Ziegler, Op. Express 13, 6296-6304 (2005)

7. L. J. Allen, W. McBride, N.L. O’Leary, and M. P. Oxley, Ultramicroscopy 100, 91-104 (2004)

8. W. K. Hsieh, F.R. Chen, J. J .Kai, A.I. Kirkland, Ultramicroscopy 98, 99-114 (2004)

9. S. C. Mayo, P.R. Miller, S.W. Wilkins, T.J. Davis, D. Gao, T. E. Gureyev, D. Paganin, D. J. Parry, A. Pogany and A. W. Stevenson, Journal of Microscopy, 207, 79-96 (2002)

10. T.E. Gureyev, A. Pogany, D. M. Paganin and S.W. Wilkins, Optics Communications 231, 53-70 (2004)

11. A. G. Peele, F. DeCarlo, P.J. Mahon, B.B. Dhal and K.A. Nugent, Rev. Sci. Instr. 76, 083707 (2005)

12. T. E. Gureyev and K. A. Nugent, Journal of the Optical Society of America A-Optics Image Science and Vision 13, 1670$1682(1996)$

13. P. Cloetens, W. Ludwig, J. Baruchel, D. Van Dyck, J. Van Landuyt, J.P. Guigay and M. Schlenker, Appl. Phys. Letters 75, 2912-2914 (1999)

14. A.V. Bronnikov, Optics Communications, 171(4-6), 239-244 (1999)

15. A.V. Bronnikov, J. of the Opt. Soc.of America A 19 (3), 472-480 (2002).

16. A. Groso, M. Stampanoni, R. Abela, P. Schneider, S. Linga and R. Müller, Appl. Phys. Lett. (2006) (to be published)

17. A. Groso, R. Abela, C. David, F. Pfeiffer and M. Stampanoni, submitted 